\title{
Axonemal Localization of the Dynein Component DNAH5 Is Not Altered in Secondary Ciliary Dyskinesia
}

\author{
HEIKE OLBRICH, JUDIT HORVÁTH, ANDREA FEKETE, NIKI TOMAS LOGES, KARIN STORM VAN'S GRAVESANDE, \\ ANDREAS BLUM, KARL HÖRMANN, AND HEYMUT OMRAN
}

\author{
Department of Pediatrics and Adolescent Medicine [H.Ol., J.H., A.F., N.T.L., K.S.G., H.Om.], University Hospital Freiburg, 79106 \\ Freiburg, Germany; Department of Pediatrics [A.F.], Research Group of Paediatrics and Nephrology, Hungarian Academy of Sciences of \\ Semmelweis University, 1082 Budapest, Hungary; Ear, Nose and Throat Surgery [A.B., K.H.], University Hospital Mannheim, \\ 68135 Mannheim, Germany
}

\begin{abstract}
Primary ciliary dyskinesia (PCD) is a heterogeneous genetic disorder characterized by recurrent airway infections and situs inversus in half of affected individuals. Diagnosis currently relies on demonstration of abnormal ciliary ultrastructure or altered ciliary beat. Alterations encountered in secondary ciliary dyskinesia (SCD) caused by inflammation often complicate the diagnostic workup. We have recently shown that in respiratory epithelial cells from PCD patients with outer dynein arm defects the dynein protein DNAH5 is mislocalized and either completely or partially absent from the ciliary axoneme. In this study, we addressed the question whether SCD might affect axonemal DNAH5 localization in respiratory cells. To induce SCD in vitro, we treated primary human respiratory epithelial cell cultures with interleukin-13 (IL-13). Ciliary function and ultrastructure were assessed by high-speed videomicroscopy and transmission electron microscopy, respectively. For in vivo localization of DNAH5, we performed nasal brushing biopsies in patients with evidence of SCD. Expression of DNAH5 was analyzed by immunofluorescence microscopy. IL-13-treated cells showed evidence of SCD. Ciliary beat frequency was significantly reduced and ultrastructural analyses showed axonemal disorganization compared with control cells. High-resolution immunofluorescence studies of respiratory epithelial cells with SCD identified in vitro and in vivo normal axonemal DNAH5 localization. DNAH5 localization is not altered by SCD, indicating a high potential for immunofluorescence analysis as a novel diagnostic tool in PCD. (Pediatr Res 59: 418422, 2006)
\end{abstract}

$\mathrm{P}_{\mathrm{t}}^{\mathrm{c}}$ CD (MIM 242650) is a rare genetic disorder that is usually transmitted as an autosomal recessive disease trait (1). Half of the affected PCD patients exhibit situs inversus due to randomization of left/right body asymmetry, which is also called Kartagener's syndrome. Recurrent airway infections result from an inborn defect of motile cilia covering epithelial cells lining the upper and lower airways responsible for mucociliary clearance of the respiratory tract. The airway disease might result in permanent lung damage like bronchiectasis, which can progress to lung failure. The aim of the

Received July 19, 2005; accepted October 31, 2005.

Correspondence: Heymut Omran, M.D., Department of Pediatrics and Adolescent Medicine, Mathildenstr. 1, 79106 Freiburg, Germany; e-mail: omran@kikli.ukl.unifreiburg.de.

This work was supported by grant P-LS-AL/7 from the Landesstiftung BadenWürttemberg.

DOI: $10.1203 / 01 . p d r .0000200809 .21364 . e 2$ therapy in PCD is prevention of permanent lung damage. However, diagnosis often is delayed because the diagnostic workup is complicated (2).

Currently, diagnosis of PCD relies on demonstration of abnormal ciliary function and/or ultrastructure in patients with recurrent airway infections with or without situs inversus (3). To analyze ciliary function, respiratory epithelial cells are usually obtained by noninvasive nasal brushing/curettage biopsy. Assessment of cilia beat pattern and frequency is performed immediately after cell sampling, preferably by the use of a high-speed videomicroscopy system. Unfortunately, this method can be only performed in a few experienced centers. In addition, alterations observed in SCD due to infections or other inflammatory processes such as allergic rhinitis might mimic PCD and result in immotile or dyskinetic cilia (4). Conversely, PCD patients often exhibit findings of secondary ciliary damage that hide the typical findings of primary defects. Characteristic ultrastructural defects that most frequently involve dynein arm composition can be detected by electron microscopy in PCD patients. However, not all ultrastructural defects encountered in PCD, especially inner dynein arm aberrations, can be securely visualized by electron microscopy (5). In addition, secondary alterations might also reduce the amount of intact cilia that can be analyzed. SCD (i.e. ciliary aplasia, axonemal membrane changes) that complicate PCD diagnosis is absent, when respiratory epithelial cells are cultured and ciliogenesis is induced in a culture system (6). However, this method is not widely available and considerable amounts of respiratory cells are necessary, which explains why this method is usually performed with material removed by ear, nose, and throat surgery.

Recent genetic studies have identified three PCD genes (DNAH5, DNAII, DNAH11) that all encode heavy and intermediate chains of the outer dynein arms (7-9). However, because of the high degree of genetic heterogeneity, DNA

Abbreviations: IL-13, interleukin-13; PCD, primary ciliary dyskinesia; SCD, secondary ciliary dyskinesia 
analysis currently cannot be used to establish PCD diagnosis on a routine basis. To possibly implement novel diagnostic strategies in a PCD diagnosis, we studied the molecular pathology of PCD on the cellular level. By high-resolution immunofluorescence imaging, we have recently shown that mutations in DNAH5 and DNAI1 cause mislocalization of the outer dynein arm component DNAH5 in respiratory epithelial cells from PCD patients (10). Investigation of nasal brushing biopsies from a large cohort of PCD patients revealed that in all PCD individuals with outer dynein arm defects, the DNAH5 protein was absent from either the entire length or only the distal part of the ciliary axonemes and instead accumulated at the ciliary base. These findings are consistent with the concept that the distinct outer dynein arm precursors are first preassembled within the cytoplasm and are then targeted to the ciliary compartment where they specifically assemble at their destined axonemal segments. Highresolution immunofluorescence imaging showed normal DNAH5 localization in healthy controls and patients with cystic fibrosis. These findings indicate that immunofluorescence analysis of respiratory epithelial cells can be used as a diagnostic tool for PCD.

To confirm that axonemal localization of the outer dynein arm component DNAH5 is not altered by SCD, we studied DNAH5 localization in vitro in an IL-13-induced SCD model and in vivo in respiratory epithelial cells from patients with evidence for SCD. Our results demonstrate that axonemal localization of DNAH5 is not altered by secondary ciliary abnormalities. These findings indicate a high potential for immunofluorescence analysis of respiratory epithelial cells as a novel diagnostic tool in PCD.

\section{METHODS}

Primary respiratory cell cultures and ciliogenesis. After informed consent using protocols approved by the Institutional Ethics Review Board at the University of Freiburg and collaborating institutions, we obtained respiratory epithelial cells from nasal conchae or polyps from patients that underwent ear, nose, and throat (ENT) surgery. To generate the IL-13-induced SCD model, we used a modified protocol reported recently (11). The removed tissue was washed with saline and epithelial cells were dissociated by incubation with $0.1 \%$ filtered pronase (protease XIV, Sigma Chemical Co.-Aldrich, Taufkirchen, Germany) overnight at $4^{\circ} \mathrm{C}$ in $10 \mathrm{~mL}$ Ham's F12-DMEM $1 / 1$ (Invitrogen, Karlsruhe, Germany). The cell suspension was washed three times and resuspended in $5 \mathrm{~mL}$ of medium containing $2 \%$ Ultroser $\mathrm{G}$ (Cytogen GmbH, Sinn, Germany) and incubated for $1 \mathrm{~h}$ in an uncoated T25 culture flask at $37^{\circ} \mathrm{C}, 5 \% \mathrm{CO}_{2}$. The cells were plated on collagen-coated tissue flasks and medium was replaced three times per week. After $\sim 3$ wk, cells reached confluency. With collagenase type IV $(200 \mathrm{U} / \mathrm{mL}$; Worthington Biochemical Corporation, St. Katharinen, Germany), the collagen gel was resolved and the cell sheet was disintegrated with a cell scraper. After washing, the cells were resuspended in $10 \mathrm{~mL}$ of medium (as described above) and cultured in uncoated T25 culture flasks. To establish suspension cultures, the flask was placed on a rotary shaker $(80 \mathrm{rpm})$ and incubated at $37^{\circ} \mathrm{C}$ for $10 \mathrm{~d}$. After $24 \mathrm{~h}$, the composition of the medium was changed to Ham's F12-DMEM 1/1 supplemented with 10\% NU-Serum (Schubert and Weiss $\mathrm{GmbH}$, München, Germany) and replacement was carried out every second day. The cells formed stable aggregates (spheroids). After $10 \mathrm{~d}$, the flasks were placed in an incubator $\left(37^{\circ} \mathrm{C}, 5 \% \mathrm{CO}_{2}\right)$.

At d 10 of suspension culture, IL-13 (Sigma Chemical Co.) at a concentration of $10 \mathrm{ng} / \mathrm{mL}$ was added to the medium in half of the culture flasks originating from two individuals. IL-13 was added to the medium for each medium exchange every second day. Thus, IL-13 exposure was done for $11 \mathrm{~d}$. After $\sim 3$ wk of suspension culture, spheroids were covered with motile cilia and cells were processed for high-speed videomicroscopy, electron microscopy, and immunofluorescence analysis.
Assessment of ciliary function by high-speed videomicroscopy. Ciliary beat frequency was determined with the SAVA system (12). Respiratory epithelial cells were analyzed with an Olympus IMT-2 microscope (40X phase contrast objective) equipped with a Redlake ES-310 Turbo monochrome high-speed video camera (Redlake, San Diego, CA) set at 125 frames per second. The ciliary beat pattern was evaluated on slow-motion playbacks.

Transmission electron microscopy. The spheroids were fixed in $2.5 \%$ glutaraldehyde in $0.1 \mathrm{~mol} / \mathrm{L}$ sodium cacodylate buffer at $4^{\circ} \mathrm{C}$, washed overnight, and postfixed in $1 \%$ osmium tetroxide. After dehydration, the samples were embedded in a propylene oxide/epoxy resin mixture. After polymerization, several resin sections were cut at approximately $100 \mathrm{~nm}$ using an ultra-microtome. The sections were picked up onto 300-mesh, thin bar copper grids. The sections were stained with Reynold's lead citrate. Transmission electron microscopy was performed with a Zeiss EM 900.

Nasal brushing biopsies from patients with SCD. We analyzed respiratory epithelial cells of ten individuals with secondary ciliary alterations caused by current upper airway disease (rhinitis or bronchitis). All patients had recurrent respiratory tract infections and exhibited at least once normal ciliary function documented by high-speed videomicroscopy. Cells were processed for highspeed videomicroscopy and immunofluorescence analysis. As a control, we used cells obtained from the PCD patient F661 with outer dynein arm defects carrying heterozygous DNAH5 mutations (R1454Q + 2970SfsX7), reported previously (8).

High-resolution immunofluorescence analysis. Samples from cultures and five nasal brushings were dripped onto glass slides, air dried, and stored at $-80^{\circ} \mathrm{C}$ until use. Cells were treated with $4 \%$ paraformaldehyde, $0.2 \%$ Triton-X 100 , and $0.5 \%$ skim milk $\left(4^{\circ} \mathrm{C}\right.$, overnight $)$ before incubation with primary (at least $2 \mathrm{~h}$ ) and secondary ( $30 \mathrm{~min}$ ) antibodies at room temperature. Appropriate controls were performed omitting the primary antibodies. The primary antibody against acetylated $\alpha$-tubulin is commercially available (mouse; Sigma Chemical Co., Taufkirchen, Germany). The antibody (rabbit) against the outer dynein arm heavy chain 5 (DNAH5) was reported previously (10). Secondary antibodies (anti-mouse Alexa Fluor 488 and anti-rabbit Alexa Fluor 546) were from Molecular Probes (Invitrogen). DNA was stained with Hoechst 33342 (Sigma Chemical Co.). Confocal images were taken on a Zeiss Axiovert 200M/LSM 510.

\section{RESULTS}

IL-13 induces SCD in human respiratory cell cultures. To study in vitro the influence of alterations observed in SCD for axonemal DNAH5 localization, we used an IL-13-induced SCD model. Quantitative analysis by high-speed videomicroscopy demonstrated that IL-13-treated respiratory epithelial cells showed in comparison to control cells a significant reduction of the ciliary beat frequency in two independent experiments using cells from two different individuals. The experiments were performed at room temperature and mean ciliary beat frequencies observed in the two studied probands ranged from 7.4 to $6.5 \mathrm{~Hz}$. In the first experiment, the mean ciliary beat frequency was reduced from $7.4 \mathrm{~Hz}$ in control cells to $6.5 \mathrm{~Hz}(12.2 \%)$ in IL-13-treated cells $(p<0.0001$, $t$ test). Whereas the mean ciliary beat frequency in untreated cells of the second proband was reduced from $6.5 \mathrm{~Hz}$ to $5.7 \mathrm{~Hz}$ $(12.3 \%)$ in IL-13-treated cells $(p<0.0001, t$ test). Thus, we found a significant reduction of the ciliary beat frequency of $\sim 12 \%$, which approximates findings reported previously (11). Qualitative analysis of slow-motion playbacks showed that most cilia exhibited normal beating patterns. However, some cilia of IL-13-treated spheroids showed a dyskinetic beating pattern and others were immotile, consistent with SCD. In contrast, cilia of control spheroids did not exhibit abnormal cilia function (see supplementary video files).

Transmission electron microscopy of control cells revealed normal anatomy of respiratory cilia (data not shown). However, in IL-13-treated cells, we identified membrane alterations comprising membrane blebs, which are the most com- 
mon secondary ciliary alterations (4). In some of these axonemal blebs, we observed excess of axonemal membrane (Fig. 1). We found no membrane alterations in control cells. Ultrastructural abnormalities affecting dynein arm composition were not noted (Fig. 1).

In summary, IL-13-treated primary respiratory cells exhibited typical functional and ultrastructural alterations indicative of SCD, consistent with findings reported previously (11).

Axonemal DNAH5 localization is not altered in SCD. We have previously reported that in patients with deficiency of outer dynein arm components, the DNAH5 protein is either completely absent or absent from the distal ciliary axoneme and instead accumulates at the ciliary base (10). To investigate whether axonemal DNAH5 localization is altered in SCD, we carefully analyzed DNAH5 localization by high-resolution immunofluorescence microscopy in the IL-13-induced in vitro SCD model. Staining of control spheroids showed that all spheroid cells facing to the outside were covered densely with cilia, which stained positive for the cilia marker acetylated $\alpha$-tubulin. DNAH5 was present throughout the ciliary axoneme and colocalized with acetylated $\alpha$-tubulin (Fig. $2 A$, see supplementary video file). Careful analysis of IL-13treated spheroids showed in all analyzed ciliated cells that DNAH5 was present throughout the ciliary axoneme independent of the size of the cilia (Fig. $2 B$, see supplementary video file).

In a next step, we analyzed samples obtained by nasal brushings from 10 patients with current airway disease. SCD was diagnosed by typical findings by high-speed videomicroscopic analysis and comprised increased numbers of leukocytes and increased frequency of partial and complete loss of

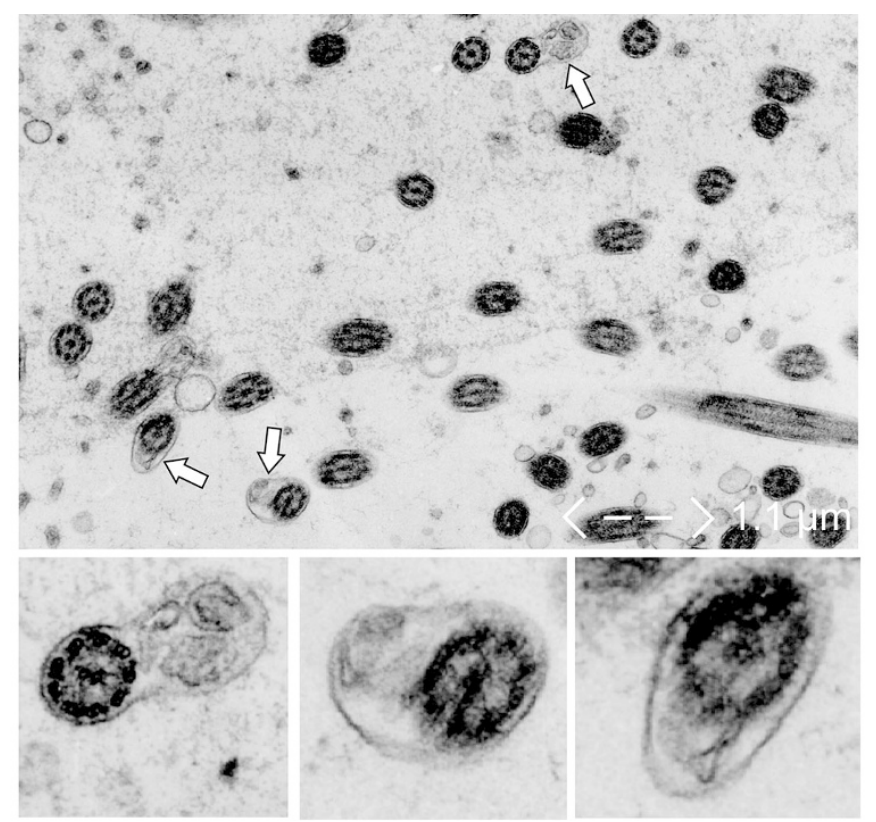

Figure 1. Transmission electron microscopy revealed axonemal membrane alterations in IL-13-treated respiratory epithelial cells. (Top) A lower magnification photograph depicting several ciliary axonemes with axonemal blebs is shown. (Bottom) Higher magnifications of three axonemal transsections (arrow) with axonemal blebs containing excess of axonemal membranes. Note that dynein arm composition is not affected (bottom left) (original magnification $\times 7450$ )
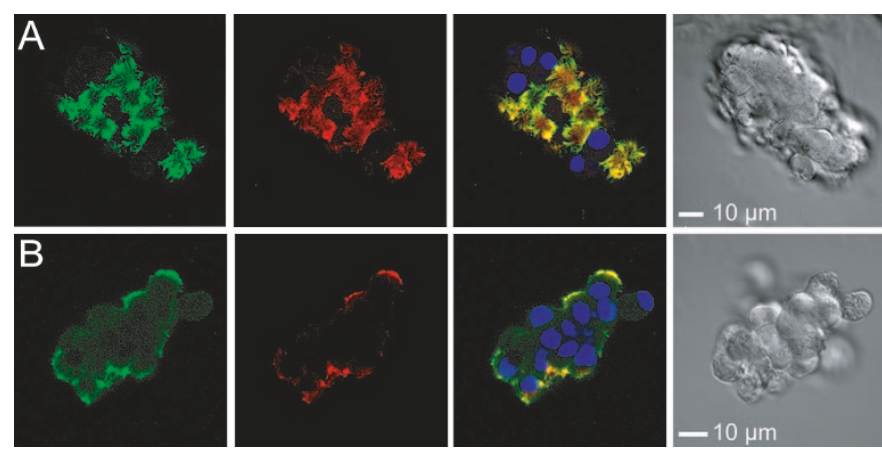

Figure 2. DNAH5 localization in respiratory cells is not altered in an IL-13-induced in vitro SCD model. Control $(A)$ and IL-13 stimulated respiratory cells $(B)$ after $21 \mathrm{~d}$ of suspension culture form stable cell aggregates (spheroids) covered with cilia. Epithelial cells were labeled with antibodies directed against acetylated $\alpha$-tubulin (green), which is a marker of the ciliary axoneme and the axonemal dynein protein DNAH5 (red), and nucleus staining (blue). Colocalization of both proteins within the ciliary axoneme is shown by the yellow color in the overlay. Photograph of phase contrast microscopy (right) (original magnification $\times 630$ ).

cilia in respiratory epithelial cells. Functional analysis revealed a mixed picture ranging from normal ciliary movement in single cells to severely dyskinetic or immotile cilia. Highresolution immunofluorescence analyses identified many respiratory cells with partial or complete loss of cilia consistent with secondary ciliary alterations. However, careful analysis of remaining ciliated cells identified in all analyzed ciliary axonemes normal axonemal content for DNAH5 and acetylated $\alpha$-tubulin (Fig. 3B). For comparison, we show abnormal DNAH5 localization in respiratory epithelial cells from a patient with PCD and outer dynein arm defects with unknown mutational status (Fig. 3C). In these cells, DNAH5 is absent from the ciliary axoneme and instead accumulates at the ciliary base. In addition, we analyzed respiratory cells during an upper respiratory tract infection from a PCD patient with compound heterozygous DNAH5 mutations (R1454Q + [2970SfsX7), as reported previously (8). Many of the respiratory cells showed an absence of cilia or membrane alterations (Fig. 3D). In the remaining ciliated respiratory cells, mutant DNAH5 was absent from the ciliary axonemes and instead localized to the basal body region (Fig. 3D).

We often noted perinuclear DNAH5 staining in cells with primary or SCD. However, we also detected this perinuclear staining occasionally in healthy control cells as reported previously (10).

\section{DISCUSSION}

Acquired ciliary abnormalities summarized under the term SCD often complicate diagnostic efforts in PCD. Currently two approaches are widely used to establish a diagnosis in PCD. Both strategies, transmission electron microscopy and direct evaluation of ciliary function by light microscopy, can be perturbed by SCD changes $(4,13)$. The aim of our study was to confirm that ciliary localization of the axonemal dynein motor protein DNAH5, which was found to be altered in PCD patients with outer dynein arm defects, is not influenced by SCD alterations. To address this question, we decided to systematically investigate DNAH5 localization in an in vitro 

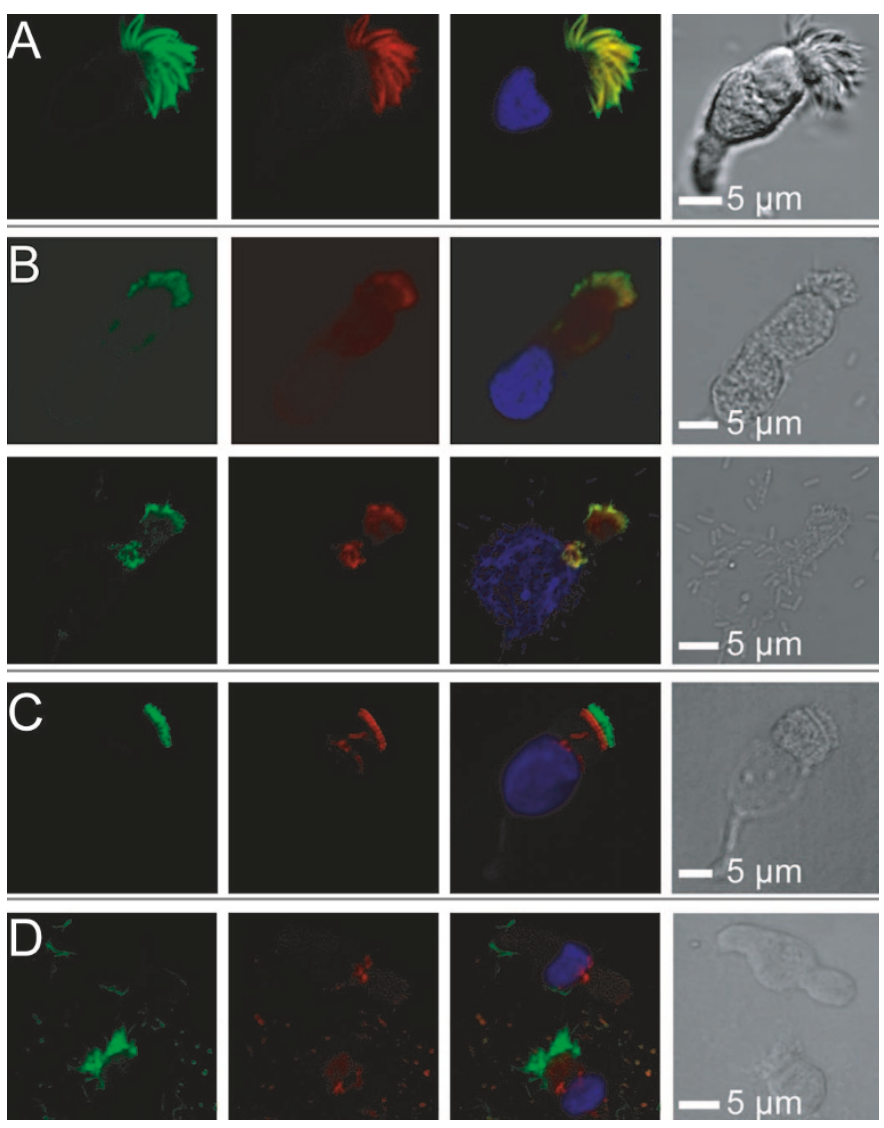

Figure 3. Axonemal DNAH5 localization is not altered in respiratory cells of patients with SCD. Epithelial cells obtained by nasal brushing were labeled with antibodies directed against acetylated $\alpha$-tubulin (green), which is a marker of the ciliary axoneme and the axonemal dynein protein DNAH5 (red), and nucleus staining (blue). The photograph on the right of each panel shows results of phase contrast microscopy. Colocalization of both proteins within the ciliary axoneme is shown by the yellow color in the overlay in cells from a healthy control $(A)$ and an individual with SCD $(B)$. Note that cilia of the patient with SCD $(B)$ are shortened (upper panel) or almost completely lacking (lower panel). Phase contrast microscopy shows bacteria and altered cells. In contrast, in cells originating from a patient with PCD and outer dynein arm defect with unknown mutational status, DNAH5 is absent from the ciliary axoneme and instead accumulates at the ciliary base $(C)$. In a PCD patient carrying compound heterozygous DNAH5 mutations (R1454Q + 2970SfsX7) and evidence of secondary respiratory cell alterations (upper cell), mutant DNAH5 is expressed and mislocalized in the remaining ciliated cells (lower cell) $(D)$. Note that the perinuclear staining does not differ between cells with primary or SCD and can be occasionally found in control cells (not shown) (original magnification $\times 630$ ).

SCD model and in respiratory cells originating from nasal brushings from patients with evidence of SCD.

Recently, it was shown that IL-13 alters mucociliary differentiation and ciliary beating of human respiratory epithelial cells (11). Based on these findings, we considered IL-13treated primary human respiratory epithelial cells an excellent in vitro SCD model. Evaluation of cilia function revealed typical SCD changes comprising reduction of ciliary beat frequency and increased number of dyskinetic and immotile cilia. Consistent with these functional defects, we identified ultrastructural changes (Fig. 1), which are commonly encountered in SCD such as axonemal membrane alterations (13-16). Thus, our findings confirm that indeed IL-13 negatively affects ciliogenesis and produces a phenotype in suspension cultures of primary human respiratory epithelial cells that resembles SCD. The data are consistent with the current concept that in asthma, increased cytokine expression not only contributes to increased mucus production but also alters ciliogenesis (11). SCD in asthmatic patients might result in decreased ability of airway clearance and allow for longer allergen exposure maintaining the allergy process.

We used the IL-13-induced SCD model to systematically analyze the subcellular localization of the outer dynein arm component DNAH5. Detailed analysis of spheroid cells by high-resolution immunofluorescence analysis with antibodies directed against acetylated $\alpha$-tubulin and DNAH5 confirmed that IL-13-treated respiratory epithelial cells exhibited normal DNAH5 staining of the ciliary axoneme (Fig. 2). Thus, we did not find any evidence that IL-13-induced SCD influences the pattern of axonemal DNAH5 localization. Analyzing respiratory epithelial cells from patients with SCD, we demonstrated that in vivo localization of DNAH5 is also not altered by SCD. We conclude that axonemal localization of the outer dynein arm component is not altered by SCD. This finding is consistent with a previous report demonstrating identical ultrastructural dynein arm composition in patients with SCD before and after ciliogenesis (6).

We have recently shown that genetic defects responsible for PCD with outer dynein arm defects cause mislocalization of DNAH5 with complete or distal absence from the ciliary axoneme $(8,10)$. We confirmed DNAH5 mislocalization in a PCD patient with an outer dynein arm defect and unknown mutational status (Fig. 3C). In addition, we showed expression and mislocalization of mutant DNAH5 in respiratory cells of a PCD patient with compound heterozygous DNAH5 mutations (R1454Q +2970 SfsX7). This is now the fourth PCD patient carrying DNAH5 mutations in whom respiratory cells could be analyzed. In the other three PCD patients, mutant DNAH5 was also expressed and mislocalized in respiratory cells (10). As expected, DNAH5 mislocalization was not affected by evidence of secondary ciliary alterations (Fig. 3D).

Our data support that DNAH5 staining can be used to identify outer dynein arm deficiency in PCD (10), and DNAH5 localization is not altered by SCD. Future work will be directed to produce novel antibodies directed against components of other axonemal protein complexes such as inner dynein arms to possibly increase the number of defects that can be diagnosed with high-resolution immunofluorescence studies.

Acknowledgments. The authors thank R. Nitschke and S. Haxelmans, Life Imaging Center, Institute for Biology I, University Freiburg, for their excellent support with confocal microscopy. They are grateful to T. Willems and M. Jorissen, Otorhinolaryngology, Department for Human Genetics, Leuven, Belgium for help in establishing the monolayer and suspension cell culture technique of the respiratory epithelium cells in our laboratory. The authors thank J. Kalnitski and M. Krome for excellent technical assistance and R. Laszig, Department of Otorhinolaryngology, University of Freiburg, Freiburg, Germany, for supporting this study. 


\section{REFERENCES}

1. Ibanez-Tallon I, Heintz N, Omran H 2003 To beat or not to beat: roles of cilia in development and disease. Hum Mol Genet 1:27-35

2. Bush A 2000 Primary ciliary dyskinesia. Acta Otorhinolaryngol Belg 54:317-324

3. Afzelius BA 1979 The immotile-cilia syndrome and other ciliary diseases. Int Rev Exp Pathol 19:1-43

4. Bertrand B, Collet S, Eloy P, Rombaux P 2000 Secondary ciliary dyskinesia in upper respiratory tract. Acta Otorhinolaryngol Belg 54:309-316

5. Escudier E, Couprie M, Duriez B, Roudot-Thoraval F, Millepied MC, PruliereEscabasse V, Labatte L, Coste A 2002 Computer-assisted analysis helps detect inner dynein arm abnormalities. Am J Respir Crit Care Med 166:1257-1262

6. Jorissen M, Willems T, Van der Schueren B, Verbeken E 2000 Secondary ciliary dyskinesia is absent after ciliogenesis in culture. Acta Otorhinolaryngol Belg 54:333-342

7. Pennarun G, Escudier E, Chapelin C, Bridoux AM, Cacheux V, Roger G, Clement A, Goossens M, Amselem S, Duriez B 1999 Loss-of-function mutations in a human gene related to Chlamydomonas reinhardtii dynein IC78 result in primary ciliary dyskinesia. Am J Hum Genet 65:1508-1519

8. Olbrich H, Haffner K, Kispert A, Volkel A, Volz A, Sasmaz G, Reinhardt R, Hennig S, Lehrach H, Konietzko N, Zariwala M, Noone PG, Knowles M, Mitchison HM, Meeks M, Chung EM, Hildebrandt F, Sudbrak R, Omran H 2002 Mutations in DNAH5 cause primary ciliary dyskinesia and randomization of left-right asymmetry. Nat Genet 30:143-144
9. Bartoloni L, Blouin JL, Pan Y, Gehrig C, Maiti AK, Scamuffa N, Rossier C, Jorissen M, Armengot M, Meeks M, Mitchison HM, Chung EM, Delozier-Blanchet CD, Craigen WJ, Antonarakis SE 2002 Mutations in the DNAH11 (axonemal heavy chain dynein type 11) gene cause one form of situs inversus totalis and most likely primary ciliary dyskinesia. Proc Natl Acad Sci U S A 99:10282-10286

10. Fliegauf M, Olbrich H, Horvath J, Wildhaber JH, Zariwala MA, Kennedy M, Knowles MR, Omran H 2005 Mislocalization of DNAH5 and DNAH9 in respiratory cells from patients with primary ciliary dyskinesia. Am J Respir Crit Care Med 171:1343-1349

11. Laoukili J, Perret E, Willems T, Minty A, Parthoens E, Houcine O, Coste A, Jorissen M, Marano F, Caput D, Tournier F 2001 IL-13 alters mucociliary differentiation and ciliary beating of human respiratory epithelial cells. J Clin Invest 108:1817-1824

12. Sisson JH, Stoner JA, Ammons BA, Wyatt TA 2003 All-digital image capture and whole-field analysis of ciliary beat frequency. J Microsc 211:103-111

13. Willems T, Jorissen M 2000 Correlations between ciliary structure and ciliary function. Acta Otorhinolaryngol Belg 54:299-308

14. Jorissen M, Willems T 2004 The secondary nature of ciliary (dis)orientation in secondary and primary ciliary dyskinesia. Acta Otolaryngol 124:527-531

15. Rayner CF, Rutman A, Dewar A, Cole PJ, Wilson R 1995 Ciliary disorientation in patients with chronic upper respiratory tract inflammation. Am J Respir Crit Care Med 151:800-804

16. Cornillie FJ, Lauweryns JM, Corbeel L 1984 Atypical bronchial cilia in children with recurrent respiratory tract infections. A comparative ultrastructural study. Pathol Res Pract 178:595-604 